\title{
Geometric phase and $o$-mode blueshift in a chiral anisotropic medium inside a Fabry-Pérot cavity
}

\author{
Ivan V. Timofeev,${ }^{1,2, *}$ Vladimir A. Gunyakov, ${ }^{1}$ Vitaly S. Sutormin, ${ }^{1}$ Sergey A. Myslivets, ${ }^{1,3}$ Vasily G. Arkhipkin, ${ }^{1,2}$ \\ Stepan Ya. Vetrov, ${ }^{1,3}$ Wei Lee, ${ }^{4}$ and Victor Ya. Zyryanov ${ }^{1}$ \\ ${ }^{1}$ Kirensky Institute of Physics, Siberian Branch of the Russian Academy of Sciences, Krasnoyarsk 660036, Russia \\ ${ }^{2}$ Laboratory for Nonlinear Optics and Spectroscopy, Siberian Federal University, Krasnoyarsk 660041, Russia \\ ${ }^{3}$ Institute of Engineering Physics and Radio Electronics, Siberian Federal University, Krasnoyarsk 660041, Russia \\ ${ }^{4}$ Institute of Imaging and Biomedical Photonics, College of Photonics, National Chiao Tung University, Guiren District, Tainan 71150, Taiwan
}

(Received 19 September 2015; published 24 November 2015)

\begin{abstract}
Anomalous spectral shift of transmission peaks is observed in a Fabry-Pérot cavity filled with a chiral anisotropic medium. The effective refractive index value resides out of the interval between the ordinary and the extraordinary refractive indices. The spectral shift is explained by contribution of a geometric phase. The problem is solved analytically using the approximate Jones matrix method, numerically using the accurate Berreman method, and geometrically using the generalized Mauguin-Poincaré rolling cone method. The $o$-mode blueshift is measured for a 4-methoxybenzylidene-4'- $n$-butylaniline twisted-nematic layer inside the Fabry-Pérot cavity. The twist is electrically induced due to the homeoplanar-twisted configuration transition in an ionic-surfactant-doped liquid crystal layer. Experimental evidence confirms the validity of the theoretical model.
\end{abstract}

DOI: 10.1103/PhysRevE.92.052504

PACS number(s): 42.70.Df, 61.30.Gd, 42.60.Da, 42.87.Bg

\section{INTRODUCTION}

Optics of liquid crystals (LCs) is well known for fruitfulness in applications and a remarkable variety of connections between observable physical phenomena [1]. A fascinating connection can be traced between the concept of geometric phase (GP) [2], also known as a topological phase, and a number of phenomena in quantum, relativistic, classical physics [3], and, in particular, optics [4-6]. Today photonics is at the apogee of topological ideas [7,8]. On the one hand, it originates from the condensed-matter graphene idea and the concept of topological insulators [9]. On the other hand, it arises from the optical technology advance. For instance, recently the three-dimensional structure of the field of light polarizations with nontrivial topology has been directly measured [10].

GP in twisted-nematic polarization phenomena [11] has application for the design of wave fronts using PancharatnamBerry phase optical elements $[12,13]$. Remarkably, polarizational GP is independent of the total phase, so it is used to offset the frequency of a laser beam by GP modulators, adding the mechanical rotation frequency of a quarter-half-quarter-waveplate Pancharatnam device [14-16]. It permits switching by ferroelectric LC [17].

Considerable attention is attracted to the research of LC placed inside a Fabry-Pérot cavity (LC-FPC), combining small-voltage LC manipulation and high spectral resolution of the Fabry-Pérot interferometer. Fundamental photonic degrees of freedom-in transmittance [18-21], phase [22], and polarization [23] — can be efficiently controlled. Polarization control usually uses the Mauguin adiabatic waveguide mode [24] in a chiral anisotropic medium, particularly in a twisted-nematic (TN) LC layer inside the Fabry-Pérot cavity (TN-FPC) [25].

The basic method for TN-FPC calculation is the Jones formalism of complex vectors and matrices of dimension

*Corresponding author: tiv@iph.krasn.ru
2 [26]. Abelès [27,28] had introduced the Chebyshev identity for the matrix power which was successfully employed in both layered and anisotropic media [29-31]. To solve the problem, one finds an eigenwave (optical mode) which conserves its shape while propagating through the medium. A set of eigenwaves is described by the eigenvectors and the eigenvalues of the Jones matrix.

Another approach to find the eigenwaves is to solve the Riccati-like ordinary differential equation system [32-34]. Within the framework of coupled-mode theory and modal analysis, this approach is equivalent to the matrix one (see Appendix C in [35]).

The account of weak reflection waves arising in LC bulk led to a generalized Berreman formalism for matrices of dimension 4 [36-38]. This generalization is necessary in media such as a cholesteric LC [39-42] and a TN cell of small thickness and other media with a sharp spatial variation of dielectric characteristics at the wavelength scale [43-46]. However, Jones formalism gives a good approximation when the thickness of the TN cell is several times larger than the wavelength and the dielectric constant varies smoothly.

Assuming no weak reflection waves arising in the LC bulk, the TN-FPC behavior was described at high voltages [25] as well as at low voltages $[34,47]$. The connection between these two extreme cases is described in [48] and generalized in [49]. Another approach is the substitution of the multilayer medium by an effective homogeneous anisotropic plate [50]. The independent method is incorporated in [24,51] using distinct mathematical tools: group theory and phase space. Also TN-FPC can be considered as a one-dimensional photonic crystal [52-55]. The photonic crystal itself can be formed by a LC material [56,57].

The orientation model of the LC layer is required to determine its optical response. To the best of our knowledge, in a TN under electrical voltage the general orientation model cannot be derived analytically and is simulated numerically, even for the one-dimensional case [58]. In contrast to an in-plane-oriented nematic [59], TN produces optical mode coupling manifested as avoided crossing of spectral 
transmission peaks [58]. A certain way to eliminate the mode coupling using anisotropic mirrors is suggested in [60]. The original theoretical study of the apparent paradox of the mode number jump for mode coupling inside a TN-FPC is proposed in [47].

This article examines the TN-FPC sample with no electric voltage deformation that allows analytical description. The direction of the spectral shift of the transmission peaks is far from obvious. That is why a visual connection is presented for the proposed spectral shift and the GP shift. This shift observation is hindered by interplay of four optical waves of opposite directions and orthogonal polarizations. Positive feedback condition describes the total spectral shift (SS), assuming three types of wave coupling. The first type of the coupling originates from LC twist which induces a twist spectral shift (TSS). The second coupling type is produced by cavity mirror reflection which induces a reflection spectral shift (RSS). The third coupling type is produced by weak reflection waves arising in the LC bulk; in the analytical part of this research it is assumed insignificant. Previously developed theory $[34,48]$ is generalized for the account of anisotropy at mirror reflection, meaning the distinct reflection phases of $e$ and $o$-wave components. The reflection is anisotropic even when the mirror is made of an isotropic material while the cavity medium itself is anisotropic.

The experimental scheme excludes significant SS impact from parasitic factors other than TSS and RSS. The experiment confirms the theory qualitatively and quantitatively.

\section{ANALYTIC MODEL}

A FPC consists of two plane mirrors (Fig. 1). The reflecting surfaces are oriented in the $x y$ plane. A nematic is placed between the cavity mirrors. The LC director is a unit vector of predominant direction of LC molecules. Twist is the state when a nematic layer is divided into thin lamellar sublayers with the nematic director being constant inside every sublayer and rotating from sublayer to sublayer. Uniform twist with no orienting external fields is implied when the LC director rotates uniformly in the plane of sublayers along the right screw. In Fig. 1 the twist angle is $80^{\circ}$; hence, the analysis is valid for an arbitrary angle. The LC director field determines the local dielectric tensor all over the medium. The extraordinary dielectric permittivity axis is collinear to the LC director. Consider the nematic with a positive uniaxial anisotropy. The extraordinary and ordinary refractive indices (RIs) correspond to waves with slow and fast phase velocities and equal to $n_{e, o}=n \pm \delta n$.

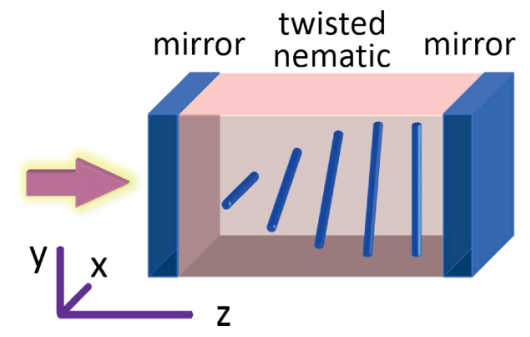

FIG. 1. (Color online) Sketch of TN-FPC, a cavity with a chiral anisotropic medium.
Let the average phase $\sigma$, anisotropy phase (angle) $\delta$, and twist angle $\varphi$ be linear functions of the coordination $z$ along the layer normal direction,

$$
\sigma(z)=n k_{0} z, \quad \delta(z)=\delta k z, \quad \varphi(z)=k_{\varphi} z,
$$

where $k_{0}=\omega / c$ is the angular wave number, $\delta k=\delta n k_{0}$, $k_{\varphi}=\varphi(L) / L$, and $L$ is the nematic layer thickness or distance between mirrors. At $z=L$, let function values $\sigma(z=L)$, $\delta(z=L)$, and $\varphi(z=L)$ be written simply as $\sigma, \delta$, and $\varphi$, respectively, without the function argument. Let the light impinge to the TN-FPC strictly in the $z$ direction. Electric field strength is described by the pair of $x$ and $y$ projections,

$$
\begin{aligned}
& E_{x}(z) \exp \{i[\omega t-\sigma(z)]\}+\text { c.c. }, \\
& E_{y}(z) \exp \{i[\omega t-\sigma(z)]\}+\text { c.c., }
\end{aligned}
$$

where c.c. stands for the complex conjugate component. It is convenient to write the pair of complex amplitudes $E_{x, y}$ as the Jones vector [26]:

$$
\vec{e}_{x y}(z)=\left[\begin{array}{c}
E_{x}(z) \\
E_{y}(z)
\end{array}\right] .
$$

The Jones matrix for an untwisted nematic at $\varphi=0$ is diagonal when the nematic director is collinear to the $x$ axis:

$$
\hat{\Delta}(\delta)=\left[\begin{array}{cc}
e^{-i \delta} & 0 \\
0 & e^{+i \delta}
\end{array}\right] .
$$

Note that the extraordinary field component along the $x$ axis has negative phase shift according to standard notion (1):

$$
\vec{e}_{x y}(L)=\hat{\Delta} \vec{e}_{x y}(0)=\left[\begin{array}{c}
E_{x}(0) e^{-i \delta} \\
E_{y}(0) e^{+i \delta}
\end{array}\right] .
$$

The Jones matrix $\hat{\Delta}$ is the transfer matrix (or propagation matrix) of the layer. It transfers a polarization state from one layer boundary to another by multiplying the corresponding Jones vector.

\section{A. Traveling eigenwaves for a uniformly twisted nematic}

For the convenience of subsequent interpretation, let us present a direct trigonometric derivation of some general expressions for a traveling wave in a chiral anisotropic medium. Let us divide a $\mathrm{TN}$ layer into a series of equal sublayers, each with the thickness $d z$ and the anisotropy angle $d \delta=\delta(d z)=\delta k d z$. Generally, the following is valid for finite not twisted sublayers. The twist angle $d \varphi=\varphi(d z)=k_{\varphi} d z$ is the angle between the dielectric permittivity main axes of neighboring sublayers. The italicized form " $d$ " is used to distinguish it from the particular case of infinitesimal differential operator.

The rotation matrix is written as

$$
\hat{\Phi}(\varphi)=\left[\begin{array}{cc}
\cos \varphi & \sin \varphi \\
-\sin \varphi & \cos \varphi
\end{array}\right] .
$$

It rotates the reference frame about the $z$ axis by the $\varphi$ angle. The Jones matrix for the wave plate (retarder) situated at the $\varphi$ angle is written as

$$
\hat{\Delta}_{\varphi}=\hat{\Phi}^{-1} \hat{\Delta} \hat{\Phi}
$$

In the rotating frame the polarization ellipse appears to be rotated by negative angle $-\varphi$. The total Jones matrix is written 
as the matrix product:

$$
\begin{aligned}
\hat{J}_{0}= & \hat{\Phi}\left(-\varphi+\frac{d \varphi}{2}\right) \hat{\Delta}(d \delta) \hat{\Phi}\left(\varphi-\frac{d \varphi}{2}\right) \cdots \\
& \times \hat{\Phi}\left(-\frac{3 d \varphi}{2}\right) \hat{\Delta}(d \delta) \hat{\Phi}\left(\frac{3 d \varphi}{2}\right) \\
& \times \hat{\Phi}\left(-\frac{d \varphi}{2}\right) \hat{\Delta}(d \delta) \hat{\Phi}\left(\frac{d \varphi}{2}\right)=\hat{\Phi}(-\varphi) \hat{J} .
\end{aligned}
$$

The product is supposed to be read from right to left with increase of $z$, because the Jones column vector is substituted on the right side of the matrix. In the rotating frame of the matrix $\hat{J}$ the LC director is always collinear to the primary axis. It is the $e-o$ frame which is often used in description of chiral media [34,61].

$z$-axis rotations are additive,

$$
\hat{\Phi}\left(\varphi_{2}\right) \hat{\Phi}\left(\varphi_{1}\right)=\hat{\Phi}\left(\varphi_{2}+\varphi_{1}\right),
$$

so the Jones matrix is naturally decomposed into the product of certain sublayer matrices,

$$
\hat{J}=d \hat{J}^{N_{S}}=\left[\hat{\Phi}\left(\frac{d \varphi}{2}\right) \hat{\Delta}(d \delta) \hat{\Phi}\left(\frac{d \varphi}{2}\right)\right]^{N_{S}},
$$

where $N_{S}=\varphi / d \varphi$ is the number of sublayers. Substitution of Eqs. (2) and (3) gives

$$
d \hat{J}=\left[\begin{array}{cc}
J_{a} & J_{b} \\
-J_{b}^{*} & J_{a}^{*}
\end{array}\right],
$$

where

$$
\begin{aligned}
J_{a} & =\cos (d \varphi) \cos (d \delta)-i \sin (d \delta), \\
J_{b} & =\sin (d \varphi) \cos (d \delta) .
\end{aligned}
$$

Eigenvectors $\vec{e}_{J}$ of the matrix $d \hat{J}$ describe polarization conserved in the rotating basis, and eigenvalues $g_{J}$ of the matrix $d \hat{J}$ are phase factors of propagation through the medium layer. The eigenvalue condition is

$$
\begin{aligned}
d \hat{J} \vec{e}_{J} & =g_{J} \vec{e}_{J}, \\
\operatorname{det}\left(d \hat{J}-g_{J} \hat{I}\right) & =0, \\
\operatorname{det}(d \hat{J})-\operatorname{tr}(d \hat{J}) g_{J}+g_{J}^{2} & =0,
\end{aligned}
$$

where $\hat{I}$ is the identity matrix. According to Eq. (5), the determinant of the matrix equals

$$
\operatorname{det}(d \hat{J})=1 .
$$

The transfer matrix is unimodular when the transferred energy is conserved. The trace of the matrix is

$$
\operatorname{tr}(d \hat{J})=2 \cos (d \varphi) \cos (d \delta) .
$$

Let us introduce a new twisted anisotropy angle defined as the following:

$$
\cos (d v) \equiv \cos (d \varphi) \cos (d \delta)
$$

The solution of Eq. (6) can then be written as

$$
g_{J}^{\mp}=\cos (d v) \mp i \sin (d v)=\exp (\mp i d v) .
$$

The eigenvectors take the forms

$$
\vec{e}_{J}^{-}=\vec{e}_{t e}=\left[\begin{array}{c}
\cos \vartheta \\
-i \sin \vartheta
\end{array}\right], \quad \vec{e}_{J}^{+}=\vec{e}_{t o}=\left[\begin{array}{c}
-i \sin \vartheta \\
\cos \vartheta
\end{array}\right],
$$

where

$$
\begin{aligned}
\vartheta & =\Theta / 2 \\
\cos \Theta & \equiv \sin (d \delta) / \sin (d v), \\
\sin \Theta & \equiv \sin (d \varphi) \cos (d \delta) / \sin (d v)
\end{aligned}
$$

Trigonometrical Eqs. (8) are equivalent to algebraic Eqs. (4.331,32) in [31].

In the literature the pair of eigenwaves described by (8) has several names [31,34,62-64]. As usual, the terminology is chosen depending on domination of the anisotropy angle or the twist angle. With zero twist $d \varphi=0$ and $\vartheta=0$, the eigenwave $\vec{e}_{J}^{-}$is simplified into an extraordinary $e$ wave, and $\vec{e}_{J}^{+}$is simplified into an ordinary $o$ wave. They are named as quasi-e and quasi-o waves [34] or te and to waves ("twisted waves") [31]. The latter italicized form is appropriate here. However, the elliptically polarized te wave may be confused with the linearly polarized TE wave (transverse electric mode), whose electric field is perpendicular to the reference plane or axis. Also, this abbreviation (extraordinary or ordinary) may be confused with the parity abbreviation (even or odd). The chirality of the te wave is opposite to LC director chirality. The chirality of the to wave is the same as LC director chirality. Consequently, the wave pair use to be termed as "opposite chirality" wave and "same chirality" wave [62]. In cholesteric LC the to wave demonstrates the Bragg reflection, while the te wave is nondiffractive. That is another way to distinguish the eigenwaves $[63,64]$.

The matrix $d \hat{J}$ is diagonalizable using the unitary matrix $\hat{U}$ to transform basic vectors $e_{e, o}$ into $e_{t e, t o}$,

$$
\begin{aligned}
\hat{U}^{-1} & =\left[\begin{array}{ll}
\vec{e}_{t e} & \vec{e}_{t o}
\end{array}\right]=\left[\begin{array}{cc}
\cos \vartheta & -i \sin \vartheta \\
-i \sin \vartheta & \cos \vartheta
\end{array}\right], \\
\hat{U} & =\left(\hat{U}^{-1}\right)^{\dagger}=\left[\begin{array}{l}
\vec{e}_{t e}^{\dagger} \\
\vec{e}_{t o}^{\dagger}
\end{array}\right]=\left[\begin{array}{cc}
\cos \vartheta & i \sin \vartheta \\
i \sin \vartheta & \cos \vartheta
\end{array}\right],
\end{aligned}
$$

where the symbol " $\nmid$ " indicates the Hermitian conjugation.

$$
d \hat{J}=\hat{U}^{-1}\left[\begin{array}{cc}
e^{-i d v} & 0 \\
0 & e^{+i d v}
\end{array}\right] \hat{U}
$$

The diagonalization simplifies the Jones matrix [Eq. (4)] exponentiation:

$$
\begin{gathered}
\hat{J}=\{d \hat{J}\}^{N_{S}}=\hat{U}^{-1}\left[\begin{array}{cc}
e^{-i v} & 0 \\
0 & e^{+i v}
\end{array}\right] \hat{U}, \\
\hat{J}=\left[\begin{array}{cc}
\cos v-i \sin v \cos \Theta & \sin \Theta \sin v \\
-\sin \Theta \sin v & \cos v+i \sin v \cos \Theta
\end{array}\right] .
\end{gathered}
$$

The uniform twist condition was used:

$$
N_{S}=\frac{\varphi}{d \varphi}=\frac{\delta}{d \delta}=\frac{v}{d v}=\frac{z}{d z} .
$$

The solution (11) is valid for sublayers of finite thickness and is the representation of Chebyshev identity [29]. Equation (7) for the angle $d v$ is the Pythagorean theorem for the spherical right triangle.

For the smooth twist function $\varphi(z)$ the sublayer thickness $d z$ tends to vanish. The solution can be simplified using the 
Pythagorean theorem for the plane right triangle:

$$
d v^{2}=d \delta^{2}+d \varphi^{2}
$$

It is easy to derive it by tailoring the cosines in Eq. (7). Multiplication by $N_{S}^{2}$ produces

$$
v^{2}=\delta^{2}+\varphi^{2} .
$$

The total wave phase then is written as

$$
\sigma \pm v=\sigma \pm \sqrt{\delta^{2}+\varphi^{2}}
$$

Let us name it the Mauguin formula. The effective RI is found by dividing both sides by $k_{0} L$ :

$$
n_{t e, t o}=n \pm \sqrt{\delta n^{2}+\left(\varphi / k_{0} L\right)^{2}} .
$$

The eigenwave ellipticity parameter $\Theta$ from Eq. (9) can be reduced as relation

$$
\tan \Theta=\varphi / \delta .
$$

Physically this ellipticity is the smoothness of the twist angle growth in comparison with the anisotropy angle growth. It is the adiabatic parameter of Mauguin's waveguide regime.

\section{B. Mirror reflection matrix}

The mirror reflection matrix has two multipliers: the phase matrix $\hat{M}_{0}$ and the half-turn rotation matrix $\hat{R}$. For a mirror made of metal with RI $n_{m}$ the reflection originates from high RI contrast with $\mathrm{LC}\left(n_{m}-n \gg \delta n\right)$. In this case the phase matrix $\hat{M}_{0}$ is approximately isotropic. However, for the dielectric multilayer Bragg mirror, the RI contrast is not high and the phase matrix is far from isotropic,

$$
\begin{aligned}
\hat{M}_{0} & =\left[\begin{array}{cc}
\exp \left(-i \mu_{e}\right) & 0 \\
0 & \exp \left(-i \mu_{o}\right)
\end{array}\right] \\
& =\exp \left(-i \sigma_{\mu}\right)\left[\begin{array}{cc}
\exp \left(-i \delta_{\mu}\right) & 0 \\
0 & \exp \left(+i \delta_{\mu}\right)
\end{array}\right] \\
& =\exp \left(-i \sigma_{\mu}\right) \hat{M} .
\end{aligned}
$$

Various algorithms are used to find phases $\mu_{e, o}$ for certain mirrors [65,66].

Assume the half-turn rotation matrix to act on the axis perpendicular to LC director,

$$
\hat{R}=\left[\begin{array}{cc}
-1 & 0 \\
0 & 1
\end{array}\right]
$$

Obviously, the double reflection of $\hat{R}^{2}$ produces the identity matrix.

\section{Perfect cavity eigenwave}

Assume the cavity is perfect (free of losses). The whole loop of the wave propagation through the cavity consists of a couple of passages and a couple of reflections. The corresponding matrix is the square of the half-loop matrix,

$$
\begin{aligned}
\hat{L} & =\left\{\hat{H} \exp \left(-i \sigma-i \sigma_{\mu}\right)\right\}^{2} \\
& =\hat{H}^{2} \exp \left(-2 i \sigma-2 i \sigma_{\mu}\right),
\end{aligned}
$$

where $\hat{H}=\hat{R} \hat{M} \hat{J}$.
If the polarization matrix $\hat{H}$ is not an identity matrix, then its eigenvectors coincide with the eigenvectors of $\hat{L}$. The eigenvalues of $\hat{L}$ are expressed through the eigenvalues of $\hat{H}$ :

$$
g_{L}=g_{H}^{2} \exp \left(-2 i \sigma-2 i \sigma_{\mu}\right) .
$$

Now one can find the eigenvalues of $\hat{H}$,

$$
\hat{H}=\left[\begin{array}{cc}
H_{a} & H_{b} \\
H_{b}^{*} & -H_{a}^{*}
\end{array}\right]
$$

where

$$
\begin{aligned}
& H_{a}=-\exp \left(-i \delta_{\mu}\right)(\cos v-i \sin v \cos \Theta), \\
& H_{b}=-\exp \left(-i \delta_{\mu}\right)(\sin \Theta \sin v),
\end{aligned}
$$

$\operatorname{det}(\hat{H})=-1$,

$$
\operatorname{tr}(\hat{H})=2 i\left(\cos v \sin \delta_{\mu}+\sin v \cos \Theta \cos \delta_{\mu}\right) \equiv 2 i \cos \rho_{0} .
$$

The eigenvalues are

$$
\begin{aligned}
g_{H} & =i\left(\cos \rho_{0} \mp i \sin \rho_{0}\right) \\
& =\exp \left[i\left(\pi / 2 \mp \rho_{0}\right)\right] \\
& \equiv \pm \exp ( \pm i \rho),
\end{aligned}
$$

where the resonator phase $\rho \equiv \pi / 2-\rho_{0}$,

$$
\sin \rho=\cos \rho_{0}=\cos v \sin \delta_{\mu}+\sin v \cos \Theta \cos \delta_{\mu} .
$$

The solution corresponds to a couple of modes resonating inside the cavity (standing waves) and let us denote them as re and $r o$ waves.

In the exotic case where $\delta_{\mu}=\pi / 2$, Eq. (17) is simplified to $\rho_{0}= \pm v$. In [60] the anisotropic mirrors are suggested with phases $\mu_{e}=\pi, \mu_{o}=0$; consequently, $\sigma_{\mu}=\delta_{\mu}=\pi / 2$,

$$
\begin{aligned}
g_{L} & =\exp \left[-2\left(\sigma+\sigma_{\mu} \pm \rho\right)\right] \\
& =\exp \left[-2\left(\sigma \mp \rho_{0}\right)+\pi(1 \pm 1)\right] \\
& =\exp [-2(\sigma \pm v)] .
\end{aligned}
$$

The reflection coupling is removed and RSS is eliminated.

In the isotropic reflection case of $\delta_{\mu}=0$, Eq. (17) reduces to

$$
\sin \rho=\sin v \cos \Theta .
$$

It is equivalent to expressions presented in $[34,48]$.

\section{Positive feedback condition}

The position of transmission peaks is determined by eigenfrequencies of perfect cavity waves (modes). They satisfy the positive feedback condition [67] for the total phase shift to be a multiple of $2 \pi$,

$$
-2 \sigma-2 \sigma_{\mu} \mp 2 \rho=-2 \pi N, \quad \mp \rho=\sigma+\sigma_{\mu}-\pi N,
$$

where $N$ is the cavity mode number. Using Eq. (17),

$$
\mp \sin (\sigma+\mu-\pi N)=\cos v \sin \delta_{\mu}+\sin v \cos \Theta \cos \delta_{\mu} \text {. }
$$

Here the minus sign corresponds to the te wave, whereas the plus sign corresponds to the to wave. It is possible to solve this trigonometric equation graphically. 
(a)

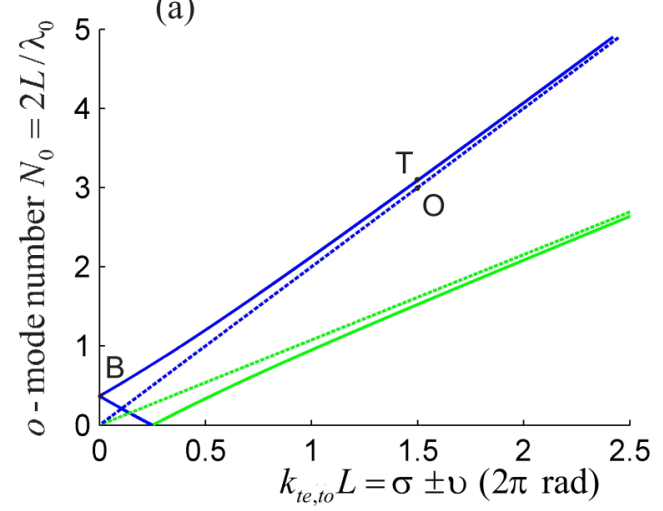

(b)

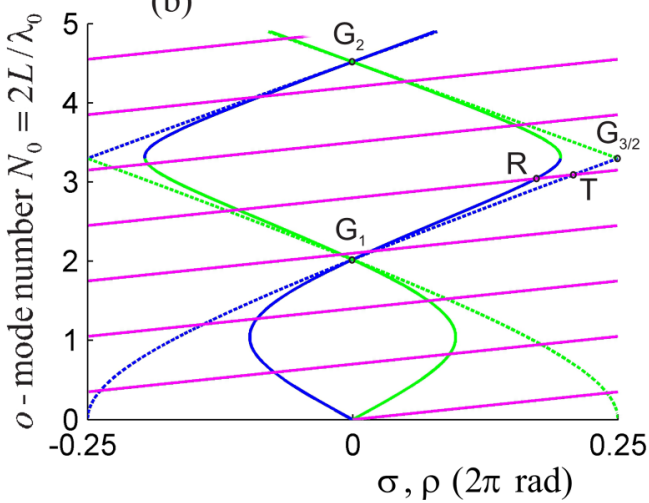

FIG. 2. (Color online) The dispersion curves. The abscissa axis shows the phase shift proportional to the wave vector. The ordinate axis shows the number of modes, proportional to the frequency of the wave. Blue (dark gray) lines show $o$, to, ro waves; green (light gray) lines show $e, t e, r e$ waves. The calculation parameters are $\varphi=\pi / 2, \delta n / n=0.3$. (a) TSS calculated with Eq. (13). Points $\mathrm{O}$ and T indicate the frequencies of mode 3 for $o$ and to waves, respectively. Point B corresponds to the zeroth mode. The splitting at point B is not shown. (b) RSS calculated with Eq. (21). Points T and R show the frequencies of mode 3 for to and ro waves, respectively. $\mathrm{G}_{1}$ and $\mathrm{G}_{2}$ are Gooch-Terry minima (22) and $\mathrm{G}_{3 / 2}$ is the Gooch-Terry maximum (23).

\section{E. Dispersion curves and TSS}

Figure 2(a) shows dispersion curves of te and to waves for $\varphi=\pi / 2$ and $\varphi=0$. Scales are dimensionless for both axes. At the ordinate the $o$-mode number $N_{o}=(\sigma-\delta) / 2 \pi=2 L / \lambda_{o}$ is proportional to the frequency of the light field. At the abscissa the phase shift is the wave number multiplied by the cavity length.

The branch of the to wave does not show the splitting by the cholesteric stop band ([1], at p. 354), for $\varphi=\pi / 2$ at $\sigma=v$, in the point B of Fig. 2(a). The splitting is omitted in Eq. (13). Dashed curves for the untwisted structure correspond to $o$ and $e$ waves: $v(\varphi=0)=\delta$. Points $\mathrm{O}$ and $\mathrm{T}$ indicate the TSS of mode 3 from the $o$ to the to wave. Calculated curves for $t e$ and to waves lie outside of the intervals of $o$ - and $e$-wave phases. It illustrates the fact that effective RI lies outside of the interval determined by the ordinary and extraordinary RI.

Let us use Eq. (11) to determine the twist phase shift,

$$
v-\delta=\sqrt{\delta^{2}+\varphi^{2}}-\delta,
$$

assuming $\varphi \ll \delta$ gives

$$
v-\delta=\delta\left(\sqrt{1+\frac{\varphi^{2}}{\delta^{2}}}-1\right) \approx \delta\left(\frac{\varphi^{2}}{2 \delta^{2}}\right)=\frac{\varphi^{2}}{2 \delta} .
$$

\section{F. Dispersion curves and RSS}

Figure 2(b) illustrates dispersion curves for $r e$ and $r o$ waves under simplification by Eq. (18):

$$
\mp \arcsin (\sin v \cos \Theta)=\sigma-\pi N \text {. }
$$

The left-hand side of Eq. (21) at $\sigma_{\mu}=0$ was shown earlier in [34] as a resonance diagram. The right-hand side of Eq. (21) produces constant-slope lines. The reduced zone with the period $\pi$ corresponds to the half-loop. The positive feedback condition (21) is fulfilled at every intersection of the magenta curve (strait lines) with the green (light gray) or blue (dark gray) one for $r e$ - and ro-wave frequencies corresponding to spectral transmission peaks. Dashed dispersion curves correspond to te and to waves $\mp \arcsin [\sin v \cos (\Theta=0)]=\mp v$.
These lines describe the zeroth-order approximation, no reflection coupling. Points $\mathrm{T}$ and $\mathrm{R}$ indicate the RSS of mode 3 from the to to the ro wave.

Dispersion curves for te, to, re, and ro waves meet at points $\mathrm{G}_{1,2, \ldots}$ where the Gooch-Terry minimum condition is valid:

$$
\sin (v)=0 .
$$

The condition was obtained for minimal TN cell transmittance [32]. In Gooch-Terry minima both resonator phase $\rho$ and twisted anisotropy phase $v$ are multiples of $\pi$. The Jones matrix $\hat{J}$ [Eq. (11)] is degenerated into a unit matrix,

$$
\hat{J}= \pm \hat{I}
$$

with \pm 1 eigenvalues and arbitrary eigenvectors. Let the condition

$$
\sin ^{2} v=1
$$

be the Gooch-Terry maximum condition. Note that this simple condition describes local maxima of the TN cell transmittance only approximately. In fact, the Gooch-Terry transmittance is a sinc function of phase, and maxima of this function are slightly nonequidistant. Figure 2(b) shows that in Gooch-Terry maxima the phase $\rho$ is maximally distant from the phase $\pm v$. This difference is given by substituting the condition (23) into Eq. (21),

$$
\begin{aligned}
v & =\arcsin (1)=\pi / 2, \\
\rho & =\arcsin (\cos \Theta)=\pi / 2-\Theta, \\
v-\rho & =\Theta .
\end{aligned}
$$

The maximum difference is described by the adiabatic parameter $\Theta$. Near the Gooch-Terry maximum the difference decreases according to the law:

$$
\min (\pi-\rho-v, v-\rho)=\frac{\sqrt{1+\Theta^{2} \tan ^{2} v}-1}{|\tan v|} .
$$

Near the Gooch-Terry maximal point $\mathrm{G}_{3 / 2}$, the four dispersion curves form a typical pattern called avoided crossing. In oscillation theory $r e$ and ro waves correspond to normal 
frequencies, while te and to waves correspond to partial frequencies [68]. In quantum mechanics re and ro waves correspond to adiabatic states, while te and to waves correspond to diabatic states [69]. Both terminologies are used in optics.

The alternation of Gooch-Terry minima and maxima in Fig. 2(b) can be interpreted as an alternation of crossings and avoided crossings of transmission peaks [47,58]. Conditions (22) and (23) for the untwisted medium correspond to the respective conditions of $\sin ^{2} \delta=0$ and $\sin ^{2} \delta=1$. The Gooch-Terry minimum matches the twisted analog of a wave plate. This wave plate retards the te wave compared to the to wave by an integer number $N_{\delta}$ of wavelengths with phase shift $2 \pi N_{\delta}$. Gooch-Terry maxima correspond to phase shifts of $\left(2 N_{\delta}+1\right) \pi$.

\section{G. Spectral shifts}

Consider the total spectral shift $\Delta \lambda$ of the TN compared with an untwisted counterpart. Without loss of generality we consider the $o$ wave. For the $e$ wave one can derive symmetric formulas with the opposite sign: $\Delta \lambda_{e}=-\Delta \lambda_{o}$

Dimensionless relative shift of the vacuum wavelength $\lambda$ is

$$
\frac{\Delta \lambda}{\lambda} \approx-\frac{\Delta k}{k_{0}-\delta k}=-\frac{\rho-\delta}{\sigma-\delta} .
$$

The average phase $\sigma$ can be excluded by use of the following relation:

$$
\frac{\lambda}{\sigma-\delta}=\frac{\lambda^{2}}{2 \pi n_{o} L}
$$

Substituting $\rho$ from Eq. (18) gives

$$
\Delta \lambda=-\frac{\lambda^{2}}{2 \pi n_{o} L}[\arcsin (\sin v \cos \Theta)-\delta] .
$$

Using approximations (24) and (20) one obtains

$$
\begin{aligned}
\Delta \lambda & =\Delta \lambda_{\mathrm{TSS}}+\Delta \lambda_{\mathrm{RSS}} \\
& =-\frac{\lambda^{2}}{2 \pi n_{o} L}\left(\frac{\varphi^{2}}{2 \delta} \mp \frac{\sqrt{1+\Theta^{2} \tan ^{2} v}-1}{\tan v}\right) .
\end{aligned}
$$

Far from the Gooch-Terry maximum condition, the second summand $\Delta \lambda_{\text {RSS }}$ can be neglected:

$$
\begin{aligned}
\Delta \lambda_{\mathrm{TSS}} & =-\frac{\lambda}{2 \pi n_{o} L}\left(\frac{\varphi^{2}}{2 \delta}\right) \\
& =-\frac{\lambda^{2} \varphi^{2}}{2 \pi n_{o} 2 L 2 \pi \delta n L / \lambda}=-\frac{\lambda^{3}}{2 n \delta n}\left(\frac{\varphi}{2 \pi L}\right)^{2} .
\end{aligned}
$$

For the $o$ wave TSS is wavelength-negative $\Delta \lambda$ TSS $<0$, and peaks are shifted in the shortwave region, i.e., blueshifted. However, near the Gooch-Terry maximum, $\tan (v) \rightarrow \infty$ so that

$$
\Delta \lambda \sim \frac{\varphi^{2}}{2 \delta}-\frac{\sqrt{1+\Theta^{2} \tan ^{2} v}-1}{|\tan v|}=\frac{\varphi^{2}}{2 \delta}-\Theta \approx \frac{\varphi^{2}-2 \varphi}{2 \delta} .
$$

For example, at $\varphi=\pi / 2$,

$$
\Delta \lambda \sim \varphi-2=\frac{\pi-4}{2}<0 .
$$

Thus, at $\varphi<2$ (in radians) the RSS component may locally reverse the total shift direction from blue to red.
TABLE I. Three levels of anisotropy complexity.

\begin{tabular}{lccc}
\hline \hline Anisotropic medium & Homogeneous & Twisted & TN-FPC \\
\hline Eigenwave & $o, e$ & to,te & ro,re \\
Eigenpolarization & Linear & Elliptic & Linear at boundaries \\
\hline \hline
\end{tabular}

\section{INTERPRETATION}

Three levels of anisotropy complexity are presented in Table I and illustrated in Fig. 3. Eigenwaves re and ro have linear polarizations at mirrors of the cavity $[47,48]$. These polarizations are biased from the LC director and the direction orthogonal to the LC director by the deflection angle $\xi$. With increasing frequency linear polarizations rotate continuously, passing from one of the principal axes to another. Therefore, we suggest to denote the eigenwaves as $e 2 o$ and $o 2 e$ waves (the English words "two" and "to" are pronounced identically). At the Gooch-Terry maximum the polarization coincides with the bisectors $\xi= \pm 45^{\circ}$. Therefore, the waves are called bisector and orthogonal bisector [48]. It is convenient to treat the $e 2 o$ wave as the $r e$ wave while it is close to the $e$ wave and to change its name to the ro wave after it passes through the bisector and gets close to an $o$ wave. Vice versa for the $o 2 e$ wave. Dispersion curves in Fig. 2(b) show this renaming by changing color at the Gooch-Terry maximum. The curve $\mathrm{G}_{1} \mathrm{RG}_{2}$ for the $o 2 e$ wave is blue (dark gray) in the lower part for the ro wave. It is green (light gray) in the upper part for the re wave.

\section{A. Poincaré sphere}

There is a variety of methods to make the results more comprehensive and visually attractive. They are the complex-number representation of polarization, Poincaré sphere [31,33,70,71], high-order and hybrid-order Poincaré spheres [72,73], admittance diagram method, Volpert-Smith chart and three-dimensional (3D) Smith chart [65,74], and the rolling cone method $[24,75]$. The last method provides a mechanical visualization of Eqs. (12) and (7) with a sum of orthogonal angular velocities of a solid cone rolling on a plane. For the sublayers of finite thickness the cone is replaced with the pyramid. The cone is rolling in a characteristic space of light polarization ellipses. This space is usually represented as a sphere of unit radius called the Poincaré sphere (PS).

Figure 4 shows a to-wave polarization trajectory and a ro-wave polarization trajectory (in blue, $\mathrm{TT}^{\prime}$, magenta, $\mathrm{RR}^{\prime}$, respectively). They smoothly evolve with the penetration depth of the TN layer under the action of operator $\hat{J}_{0}$. For the right-handed to and ro waves it is convenient to set the right-handed polarization in the upper hemisphere of PS as in $[33,35]$, and not in the lower one, as in $[6,31,76]$. The trajectory $\mathrm{RR}^{\prime}$ is the spherical trochoid [77]. It is associated with a trajectory of a point rigidly connected with a solid cone [Fig. 5(a)] rolling without slipping on a plane [24]. A stereographic projection of a similar trajectory is presented in Ref. [33], p. 136, Fig. 2.24.

For further consideration it is essential that the unimodular Jones matrix has the geometrical sense of the PS rotation. Jones vectors correspond to PS points. Distances between points are conserved at transformations on the spherical surface. The 
(a)

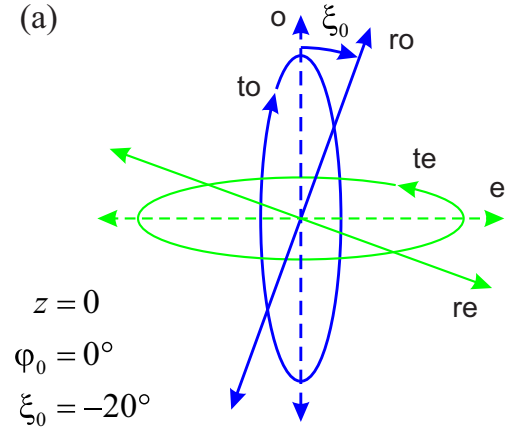

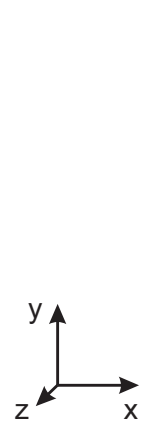

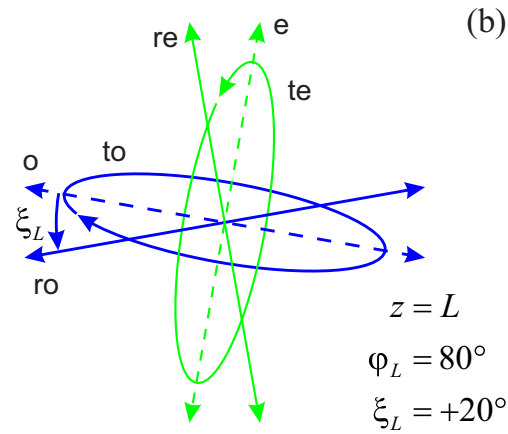

(b)

FIG. 3. (Color online) Representative polarizations at medium boundaries $z=0$ (a) and $z=L$ (b). Blue (dark gray) lines show $o$, to, ro waves; green (light gray) lines show $e, t e, r e$ waves. The twist angle $\varphi$ is between the $o$ and the $y$ directions. The $o$-deflection angle $\xi$ is between $o$ and $r o$ directions. The $e$-deflection angle between $e$ and $r e$ directions has the same magnitude as $\xi$ at both boundaries.

PS point is often regarded either as the normalized triplet of Stokes parameters or as the polarization ellipse traced by the terminal point of the field vector. In both cases PS is a 2D manifold. However, the Jones vector has three degrees of freedom. Its third phase is the temporal phase. It progresses by $2 \pi$ while the field vector passes the elliptic trajectory. The 3-phase polarization state is the one-to-one representation of the Jones vector. This polarization state is regarded either as the triplet of Euler angles or as the unit quaternion. The space of all polarization states is a 3-sphere. The Hopf fibration projects it onto PS which is a 2-sphere [6]. The unit quaternion may be imagined as a "flag" consisting of two arrows. The first polarization arrow goes from the center of PS to its surface. The second arrow of temporal phase is connected to the terminal

(a)

(b)
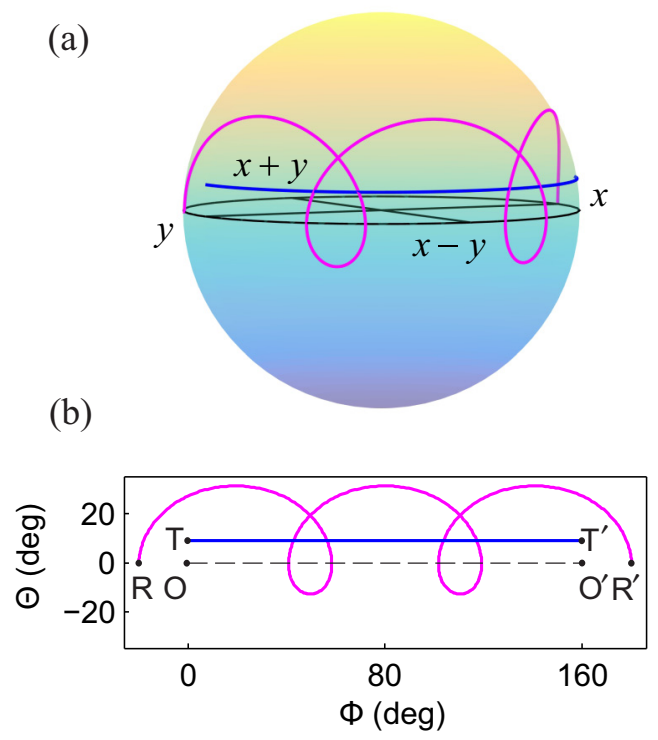

FIG. 4. (Color online) Poincaré sphere (a) and a portion of its cylindrical projection (b). Directions $y, x-y, x$, and $x+y$ correspond to angles $\Phi=2 \varphi=0^{\circ}, 90^{\circ}, 180^{\circ}$, and $270^{\circ}$, respectively. Trajectories under the action of operator $\hat{J}_{0}$ for $\varphi=80^{\circ}$ : $\mathrm{OO}^{\prime}$, linear polarizations on the PS "equator" corresponding to $o$ wave; $\mathrm{TT}^{\prime}$, to-wave trajectory on the PS "parallel” with latitude $\Theta ; \mathrm{RR}^{\prime}$, ro-wave trajectory, a spherical trochoid. The parameters correspond to the rightmost peak in the below-mentioned experimental spectrum of Fig. $8(\mathrm{a}), \lambda=579.1 \mathrm{~nm}$. point of the first arrow and goes in a perpendicular direction (similar to that shown in Fig. 2 of Ref. [78]). If the polarization arrow is rotated, then the entire flag of the polarization state is rotated about the same axis. With the temporal phase increase the second arrow rotates around the first one. Remarkably, one period on the polarization ellipse corresponds to two revolutions of the flag. Strictly speaking, Jones matrices form

(a)
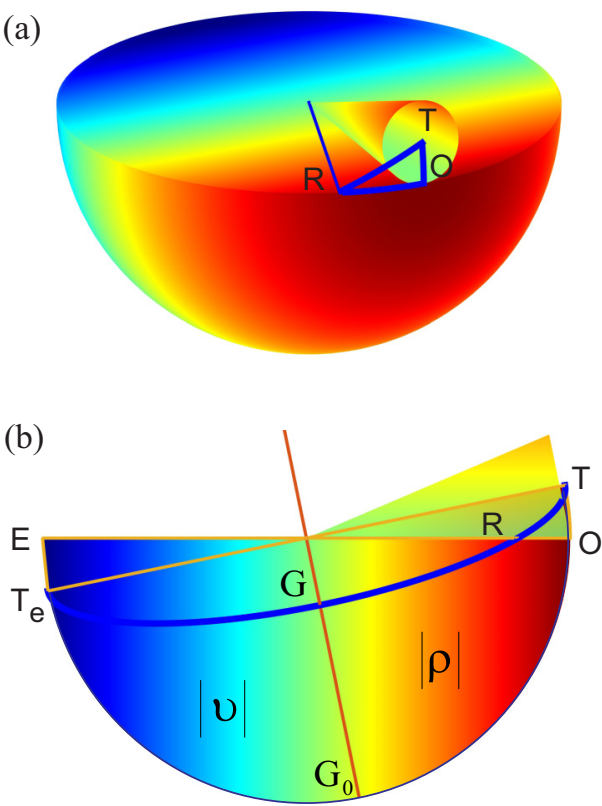

FIG. 5. (Color online) The rolling cone method on the PS. (a) Points $\mathrm{O}, \mathrm{T}$, and $\mathrm{R}$ describe the polarizations of $o$, $t o$, and $r o$ waves at the boundary of the twisted layer. The Mauguin cone contains the point $\mathrm{T}$ on its axis and the point $\mathrm{O}$ on its generator. Spherical triangle OTR has a right angle $\angle$ ROT. The cathetus $\mathrm{RO}=\Xi=2 \xi$ lies on the "equator." The cathetus TO $=\Theta=2 \vartheta$ is perpendicular to the "equator." Acute angles are $\angle \mathrm{RTO}=v$ and $\angle \mathrm{ORT}=\rho_{0}$. The area is equal to spherical excess $\Omega(\mathrm{OTR})=\left(\pi / 2+v+\rho_{0}\right)-\pi=v-\rho$. (b) Points $\mathrm{E}$ and $\mathrm{T}_{e}$ describe polarizations of $e$ and te waves. The great circle $\mathrm{G}_{0} \mathrm{G}$ is perpendicular to the diameter $\mathrm{TT}_{e}$. The big circle $\mathrm{T}_{e}$ GRT in the Gooch-Terry minimum coincides with the great circle $\mathrm{T}_{e} \mathrm{G}_{0} \mathrm{OT}$. Distance to the Gooch-Terry minimum for the to wave is determined by the phase $v=\angle \mathrm{G}_{0} \mathrm{G}=\Omega\left(\mathrm{TG}_{0} \mathrm{G}\right)$, and for $r o$ waves it is determined by the phase $\rho=\Omega\left(\mathrm{G}_{0} \mathrm{GRO}\right)$. 
the special unitary group $\mathrm{SU}(2)$. This group is the universal covering of the rotation group $\mathrm{SO}(3)$. The covering is two sheeted [79-81].

\section{B. Geometric phase}

The parallel transport of a geometric object on a curved surface rotates the object about its own axis. A classic example is the Foucault pendulum with the rotation of its swing plane caused by Earth's daily rotation. Similarly, the parallel transport of the polarization state on the PS curved surface leads to the phase shift called the GP. It is caused by global geometric characteristics, such as the curvature and the parallel transport trajectory, and independent of local characteristics, such as the speed of state movement along the trajectory. There is a geometric formula for closed trajectories. Applied to polarization it claims that GP $\beta$ is equal to minus half the area $\Omega$ encircled by the trajectory on PS:

$$
\beta=-\Omega / 2 .
$$

A rigorous proof is given in [6] using Stokes' theorem.

\section{Geometric calculation of the phase shift corresponding to TSS for traveling wave}

We apply the geometric formula to find the phase shift of the traveling wave at the trajectory $\mathrm{TT}^{\prime}$ in Fig. 4. The elliptical wave expressed by Eq. (8) is a superposition of $o$ and $e$ waves with relevant RIs. The averaged RI should be chosen in the form that takes into account the analog of the Aharonov-Anandan dynamic phase [see Ref. [6], Eq. (5.19)]:

$$
\bar{n}=n-\delta n \cos \Theta, \quad \alpha=\bar{n} k_{0} L=\sigma-\delta \cos \Theta .
$$

The minus sign corresponds to the $o$ wave. This RI normalization reduces the PS rotation to parallel transport along the great-circle trajectory.

Let the twist angle $\varphi=\pi$. Then $\Phi=2 \varphi=2 \pi$ makes one turn on the PS "parallel" with latitude $\Theta$. The area between the "equator" and this "parallel" is equal to the side surface of a cylinder of unit radius and of height equal to $\sin \Theta$ (see Ref. [82], p. 260, Lambert cylindrical equal-area projection),

$$
\Omega(\varphi=\pi)=2 \pi \sin \Theta .
$$

For an arbitrary twist angle,

$$
\Omega(\varphi)=2 \varphi \sin \Theta .
$$

The total phase shift $\gamma$ consists of the dynamic phase $\alpha$ and the GP $\beta: \gamma=\alpha+\beta$. The geometric formula (28) and equations $(29,30)$ give

$$
\gamma=\sigma-\delta \cos \Theta-\varphi \sin \Theta .
$$

Equations (12) and (15) produce a transformation $\delta=v \cos \Theta$, $\varphi=v \sin \Theta$. This transformation gives

$$
\gamma=\sigma-v\left(\cos ^{2} \Theta+\sin ^{2} \Theta\right)=\sigma-\sqrt{\delta^{2}+\varphi^{2}} .
$$

Indeed, the result coincides with the Mauguin formula (13).

\section{Geometric calculation of the phase shift corresponding to RSS for cavity wave}

The Mauguin-Poincaré rolling cone method is easily expanded to the Hopf bundle of PS. In fact, the generalization is a consequence of the solid cone analogy. This generalization makes it possible to find certain phase relations trigonometrically. The phase integration along the trajectory $\mathrm{RR}^{\prime}$ corresponds to the matrix multiplication of Eq. (4). Diagonalization of Eq. (10) is a simplifying algebraic transition to the rotating frame written as the product of three matrices. Geometrically, it corresponds to the transition from the trajectory $\mathrm{RR}^{\prime}$ to the chain of three arcs: RT-TT'- $\mathrm{T}^{\prime} \mathrm{R}^{\prime}$ [Fig. 4(b)], wherein the first and the last arcs are to be chosen as geodesics. The great-circle arc RT [see Fig. 5(a)] matches the parallel transport and the GP corresponds to the Pancharatnam phase [4] without any normalizing dynamic phase of Eq. (29).

During the passage of the distance $L$ ahead through the cavity the traveling to wave in rotating frame receives the phase shift $v$. Therefore, PS is rotated by the angle $2 v$. After the rotation the linear polarization $R$ is returned back to the "equator" at the point $\mathrm{R}^{\prime}$. It corresponds to reflection symmetry of triangles OTR and $\mathrm{O}^{\prime} \mathrm{T}^{\prime} \mathrm{R}^{\prime}$. The mirror reflection corresponds to the triangle reflection symmetry with respect to the $\mathrm{RO}$ arc (improper rotation). The resultant GP shift of the ro wave compared with that of the to wave accounts the triangle area four times per loop cycle $4 \Omega(\mathrm{OTR})=2 \beta_{\mathrm{RSs}}$.

The rolling cone method works even when the adiabatic condition is violated. In [49] the nonuniform LC twist is examined, for example, under electric voltage. However, in the symmetric case for the cone opening angle $\Theta(z)=$ $\Theta(L-z)$ the eigenwave preserves linear polarization on the layer boundary. For practice, the linear polarization of the transmitted light is advantageous, because of the reliable blocking of transmission [83].

The account of mirror phase shift $\delta_{\mu}$ by anisotropic reflection is equivalent to a wave-plate action. On PS this leads to an additional rotation of the RO arc by the angle of $\delta_{\mu}$ off the "equator." Pythagorean theorem (18) for the right spherical triangle OTR transforms to the cosine theorem (17) for the angles of the spherical triangle. The point $\mathrm{R}$ leaves the PS equator. It means that the corresponding transmission peak becomes elliptically polarized. The superposition of opposing traveling waves on a perfect cavity mirror remains linearly polarized at $\delta_{\mu} \neq 0$ only when $\sigma_{\mu}=\pi N_{\mu} / 2$ with integer $N_{\mu}$.

\section{E. Connection between dispersion curve phases and PS angles}

With increasing frequency the Mauguin cone rolling angle $2 v$ is increased uniformly. The to wave corresponds to the Mauguin cone axis on PS. The point $\mathrm{T}$ is fixed, assuming constant adiabatic parameter: $\Theta(\omega) \approx$ const. Without changing the polarization, the to wave acquires a $\pi$ phase between the adjacent Gooch-Terry minima [see the section $\mathrm{G}_{1} \mathrm{G}_{3 / 2} \mathrm{G}_{2}$ of the dispersion curve at Fig. 2(b)]. The ro wave gains the phase $\rho$ (dispersion curve section $\mathrm{G}_{1} \mathrm{RG}_{2}$ ) which is less than the phase $v$ by the area of a spherical triangle $\Omega(\mathrm{OTR})=$ $v-\rho$. During the passage of the Gooch-Terry maximum this area has a critical value $\Omega(\mathrm{OTR})=\Theta$ in accordance with Eq. (24). 
The cathetus RO $=\Xi=2 \xi$ of the triangle OTR is given by Napier's rules for right spherical triangles [81]:

$$
\tan \Xi=-\sin \Theta \tan v \text {. }
$$

This equation determines the deflection angle $\xi$ of the boundary $r o$-wave linear polarization from the LC director (Fig. 3). Figure 4(b) shows the arc TR rotating when $v$ increases. The point $\mathrm{R}$ moves nonuniformly along the PS equator. The movement is faster near the Gooch-Terry maximum. In adiabatic approximation $\Theta \ll \pi / 2$ the point $\mathrm{R}$ jumps at the Gooch-Terry maximum so that $\xi \approx 0$ for the $r o$ wave and $\xi \approx \pi / 2$ for the re wave.

Exact correspondence between optical wave phases and characteristic space angles provides a visual support and a qualitative understanding of the phenomenon supporting the validity of the obtained solution.

\section{F. Intermediate optical response presumption}

The motivation for this study was the debate about the direction of the transmission peak SS in a TN layer. The intermediate optical response presumption was formulated for an anisotropic medium whose effective RI is between the ordinary and extraordinary $R I$,

$$
n_{o}<\tilde{n}<n_{e}
$$

Here are a few abstract examples supporting the intermediate optical response presumption.

(1) The average RI of a nematic in the isotropic phase is $[1,75]$

$$
n_{i s o}^{2}=\left(n_{e}^{2}+2 n_{o}^{2}\right) / 3 .
$$

(2) In a homogeneous, uniaxially anisotropic medium, the extraordinary light wave propagating at an angle $\theta$ to the optical axis has the following RI [Ref. [1], Eq. (11.6)]:

$$
n_{e}(\theta)=n_{o} n_{e}(0) / \sqrt{n_{e}^{2}(0) \cos ^{2} \theta+n_{o}^{2} \sin ^{2} \theta} .
$$

(3) The thin sublayer of the TN layer has effective RI according to the normalization (29).

A nonadditive response of a slab of sublayers leads to the GP (28). As a result, the Mauguin formula (14) contradicts the intermediate optical response presumption (31):

$$
n_{t e, t o}=n \pm \sqrt{\delta n^{2}+\left(\varphi / k_{0} L\right)^{2}}, \quad n_{t o}<n_{o}<n_{e}<n_{t e} .
$$

The stated contradiction admits an experimental test. With an increase in the effective RI (i.e., increase in the optical length of the cavity), transmission peaks are shifted to the red. For the $o$ wave in untwisted structure the effective RI is equal to the ordinary RI. According to the intermediate optical response presumption [Eq. (31)] the twisted effective RI shifts towards the extraordinary RI; in other words, the effective RI increases. This predicts the redshift $\Delta \lambda_{\text {TSS }}>0$ for spectral peaks. In contrast, the Mauguin formula predicts the blueshift $\Delta \lambda_{\text {TSS }}<0$ (27).

\section{EXPERIMENT}

\section{A. LC orientation}

An experimental study of the shift of the $o$-polarized spectral transmission peaks to shorter wavelengths was carried out inside a LC-FPC. The Fabry-Pérot cavity consisting of two dielectric mirrors (Fig. 6, bottom row) is treated as a LC cell. It was filled with the nematic LC 4-methoxybenzylidene$4^{\prime}-n$-butylaniline (MBBA) doped with the cationic surfactant cethyltrimethyl ammonium bromide (CTAB) in the weight ratio 1:0.003. The cavity gap is $7 \mu \mathrm{m}$. CTAB molecules within MBBA dissociate into $\mathrm{Br}$ anions and surface-active CTA cations. The latter are adsorbed at the surfaces of aligning layers and, at the sufficient concentration, can form a layer specifying the homeotropic coupling condition for nematic molecules $[84,85]$.

A multilayer mirror coating consists of six layers of zirconium dioxide $\left(\mathrm{ZrO}_{2}\right)$ with $\mathrm{RI}$ of 2.04 and a thickness of $55 \mathrm{~nm}$ and five layers of silicon dioxide $\left(\mathrm{SiO}_{2}\right)$ with $\mathrm{RI}$ of 1.45 and a thickness of $102 \mathrm{~nm}$ alternately deposited on the surface of a quartz substrate. The alternating layers produce the reflection band in the range of $420-610 \mathrm{~nm}$. Thin $(\sim 150 \mathrm{~nm})$ ITO electrodes were deposited on the upper layers of $\mathrm{ZrO}_{2}$ to apply the electric field normally to the LC-FPC mirrors. Electrodes were covered with different polymeric alignment films by spin-coating to implement the initial homeoplanar director orientation [Fig. 6(a), bottom row]. The top substrate was covered with a planar-orienting film of pure polyvinyl alcohol (PVA). The bottom substrate was covered with a PVA film doped with glycerin $(\mathrm{Gl})$ compound in the weight ratio 1:0.61. With the utilized CTAB concentration the surfactant ion molecules were adsorbed on the PVA-Gl film. The formed layer shields the planar-orienting effect of the polymer coating and provides the homeotropic anchoring for MBBA. Polymer films on both dielectric mirrors were rubbed unidirectionally to define the axis of easy orientation. The angle between the rubbing directions at the top $\left(\mathrm{R}_{1}\right)$ and bottom $\left(\mathrm{R}_{2}\right)$ mirrors is $90^{\circ}$.

Inside the LC-FPC cell at constant voltage value $U=4 \mathrm{~V}$, the orientation transition from homeoplanar to twist director configuration is induced by ionic modification of surface anchoring [86]. This transition is accompanied by a modification in polarizing microscope optical texture of the LC-FPC cell under the parallel- and crossed-polarizer scheme (Fig. 6, top row and middle row, respectively). For example, under crossed polarizers the optical texture of LC-FPC cells in the initial state is uniformly dark when the $\mathrm{R}_{1}$ direction coincides with the transmission axis of the polarizer [Fig. 6(a), middle row]. The light transmission is increased when the voltage is applied and the twist-configured FPC (TN-FPC) is formed [Fig. 6(b), middle row]. However, under the parallel-polarizer scheme the TN-FPC transmittance is low [Fig. 6(b), upper row] due to the rotation of the polarization plane of linearly polarized light at an angle close to $90^{\circ}$ after passing the $\mathrm{TN}$ layer.

The experimental scheme excludes a significant impact of parasitic factors on the shift. The structure is twisted uniformly, because every sublayer has constant twist torque created by different rubbing orientations. The twist structure is under the voltage of $4 \mathrm{~V}$. However, this does not lead to any deformation of the structure. First, in the volume of the cell the voltage is 
(a)
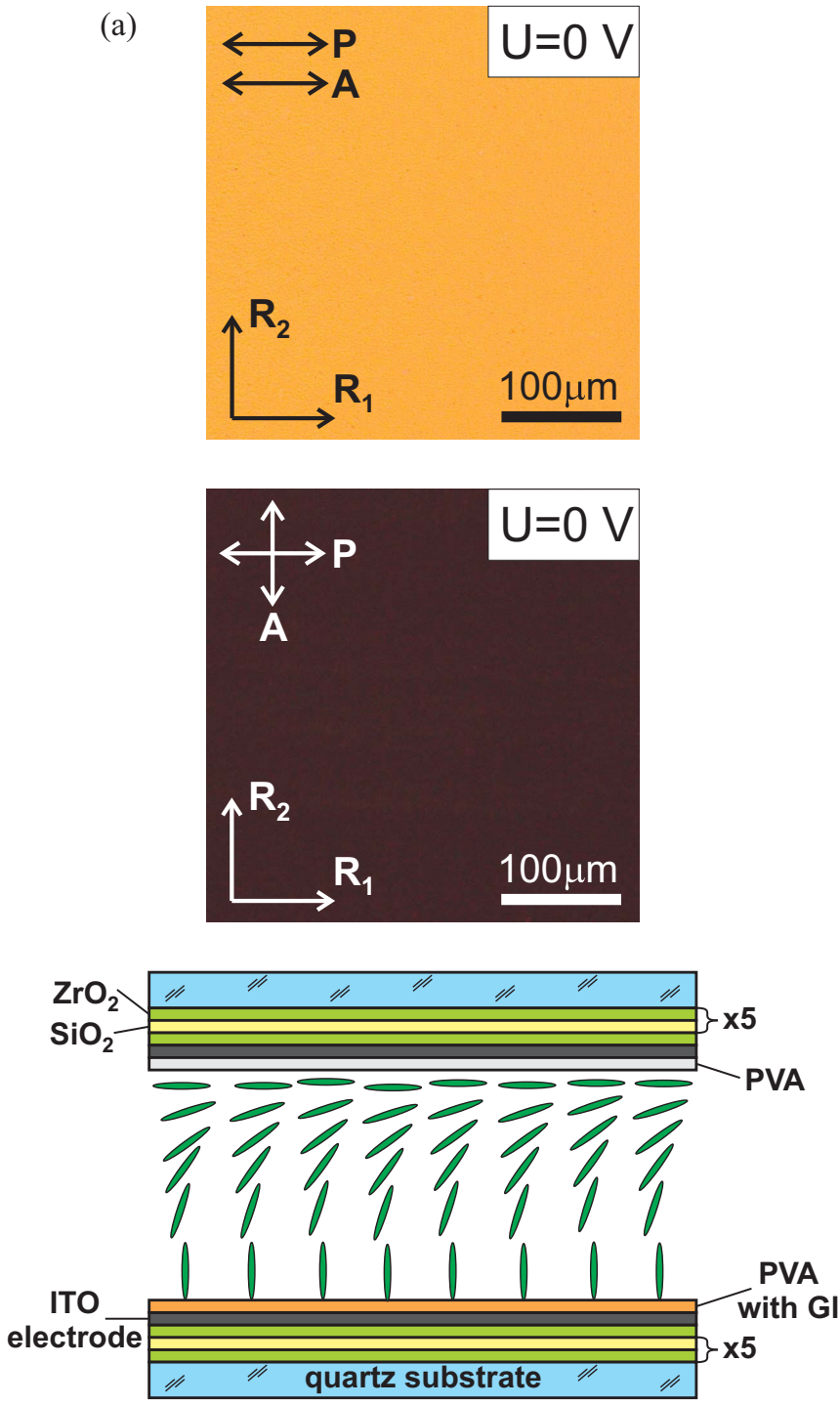

(b)
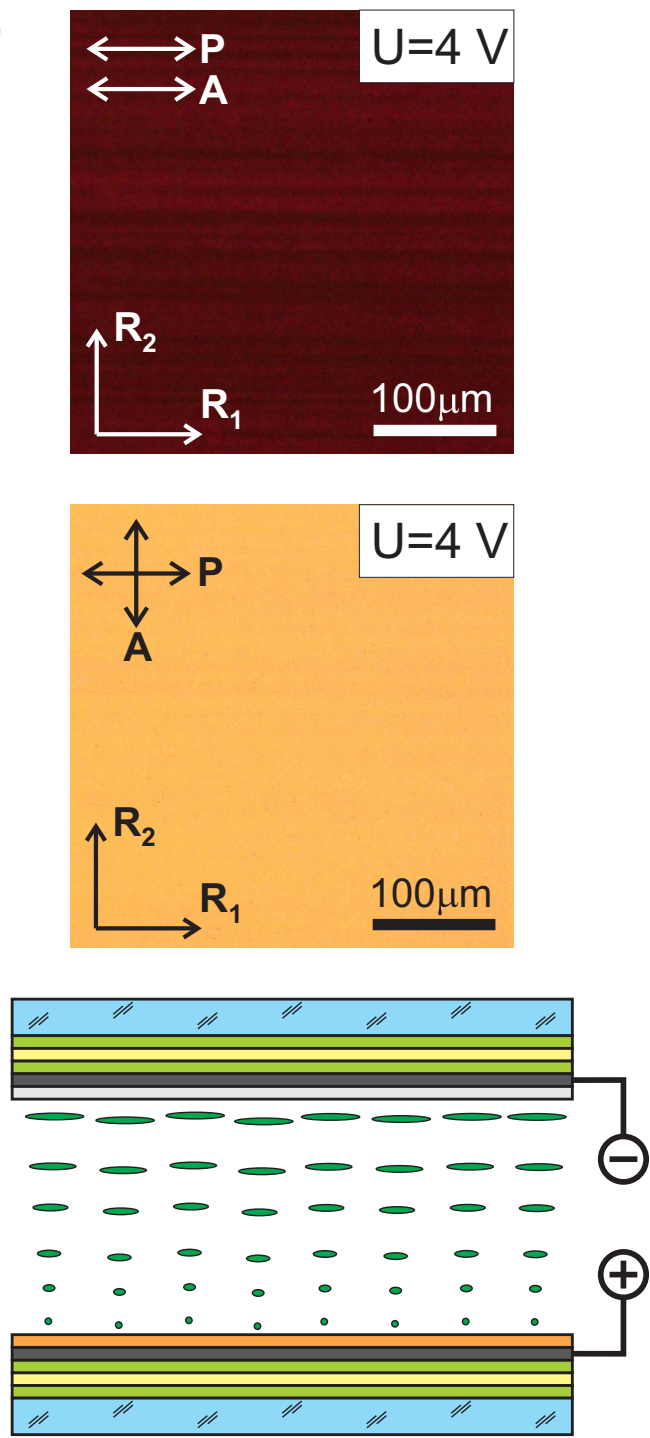

FIG. 6. (Color online) Microphotographs of optical textures of LC-FPC under the parallel-polarizer scheme (top row) and crossed polarizers (middle row) and the respective diagrams of the LC director configurations (bottom row). (a) Homeoplanar orientation at $U=0 \mathrm{~V}$; (b) twist orientation at $U=4 \mathrm{~V}$. Polarizer $(\mathrm{P})$ and analyzer (A) directions are represented by the double arrows. $\mathrm{R}_{1}$ and $\mathrm{R}_{2}$ are rubbing directions of the top and bottom mirrors, respectively.

partially compensated by surface charges. Second, MBBA is oriented transversely to electric field.

\section{B. Cavity and its spectra}

Polarized transmission spectra of LC-FPC with two different configurations of MBBA director for normally incident light were measured using an Ocean Optics HR4000 spectrometer equipped with fiber optics (Fig. 7). The LC-FPC sample was placed inside the optical channel with planar orientant $\left(\mathrm{R}_{1} \| x\right)$ at the input mirror. A Glan prism $(\mathrm{P})$ was used as a polarizing element with the linear polarization along the $y$ axis (i.e., orthogonal to the director on the input mirror). In this experimental scheme the $o$-polarized transmission spectrum was measured regardless of structural transformations in the nematic volume. Spectra were recorded at a fixed temperature of $23.0^{\circ} \mathrm{C}$. Thermal stabilization error was no more than $\pm 0.2^{\circ} \mathrm{C}$. The voltage was generated by a power supply (AKTAKOM ATH-1236).

\section{Comparison of experiment, simulation, and analytic formulas}

Three measurements were averaged to calculate the experimental SS (Fig. 8). The experimental resolution of $0.25 \mathrm{~nm}$ was improved by fitting spectral peaks by Voigt contours. The confidence interval was calculated as the corrected sample standard deviation multiplied by the Student coefficient with 95\% reliability: $t_{0.05,2}=4.3027$.

The Berreman method [37,38] was used for numerical simulations. The method evaluates polarization vectors and transfer matrices of dimension 4 to take into account the weak reflection waves arising in the LC bulk (multiple anisotropic reflections between the sublayers). The TN layer was divided into 200 sublayers and the calculated spectral resolution 


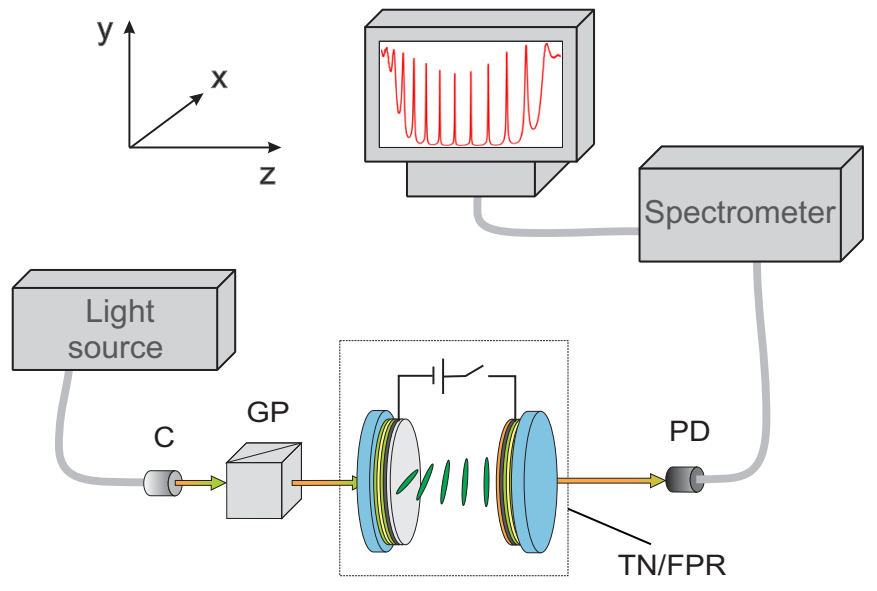

FIG. 7. (Color online) Experimental setup for measurement of polarized transmittance spectra of LC-FPC. C, fiber collimator; GP, Glan prism; PD, photodetector.

was $0.01 \mathrm{~nm}$. Some parameters were considerably tuned to match the experimental spectra. The thicknesses and RIs of amorphous layers constituting the dielectric mirror were taken for $\mathrm{SiO}_{2}, 83 \mathrm{~nm}$ and 1.45; for $\mathrm{ZrO}_{2}, 66 \mathrm{~nm}$ and 2.02; for each ITO layer, $117 \mathrm{~nm}$ and $1.88858+0.022 i$ taking the absorption
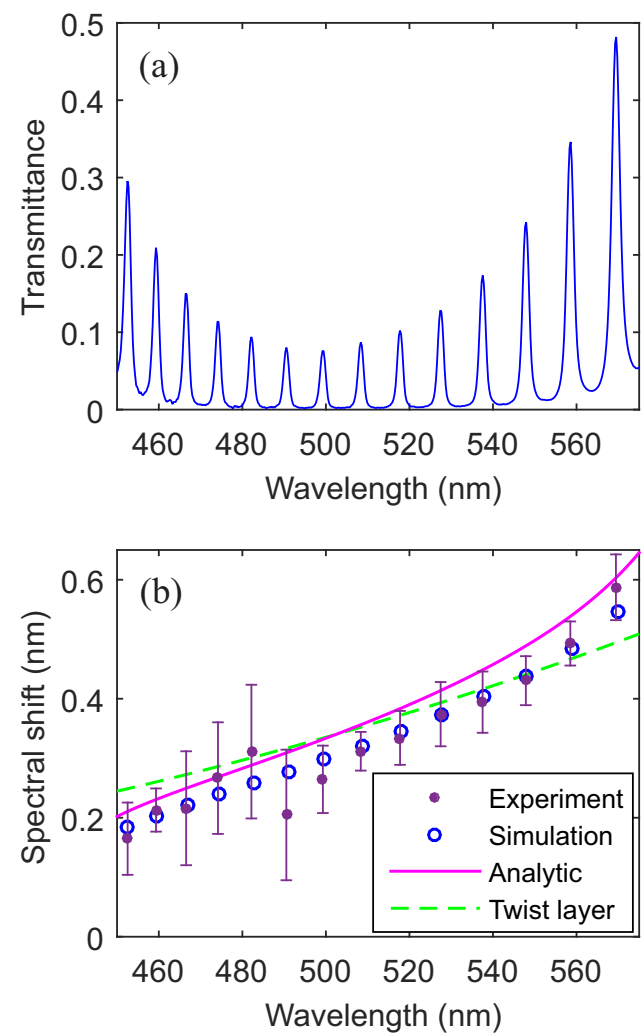

FIG. 8. (Color online) Ordinary-polarized LC-FPC spectrum (a) and the SS of transmission peaks (b). •, experimental shift values; $\circ$, calculated shift values obtained by direct numerical simulation using the Berreman method; solid curve, analytical shift calculated from Eq. (25) obtained by the Jones method; dashed curve, the shift $\Delta \lambda_{\text {TSS }}$ calculated without mirror effect from Eq. (27). The parameters of the rightmost peak with $\lambda=579.1 \mathrm{~nm}$ were taken to calculate Fig. 4 .
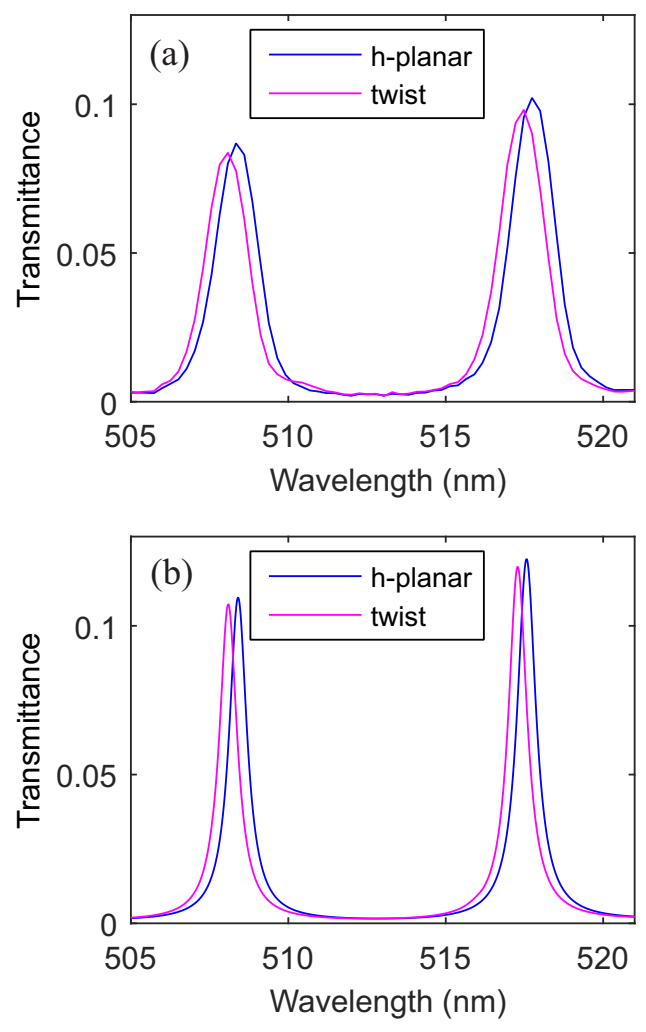

FIG. 9. (Color online) Magnified experimental (a) and calculated (b) transmittance spectral peaks for homeoplanar [blue (dark gray)] and twisted [magenta (light gray)] configurations in LC-FPC. The twist leads to shorter wavelength shifts.

into account; for the substrate RI, 1.45 and for the PVA RI, 1.515; two thicknesses of PVA layers, 300 and $600 \mathrm{~nm}$; for MBBA extraordinary RI: 1.737 and ordinary RI, 1.549, both with RI imaginary part, $0.00078 i$. The thickness of MBBA layer was $7980 \mathrm{~nm}$; the twist angle was $80^{\circ}$.

The material dispersion gave some minor changes in the spectra and the shift. The most notable changes were the absorption dispersion and the change in MBBA layer thickness by $40 \mathrm{~nm}$. Therefore, the spectra illustrated in Figs. 8 and 9 were calculated assuming no material dispersion.

Experimental transmission peaks are broadened no more than 2 times larger than the calculated peaks (Fig. 9). This was achieved by additional flatness tuning of the cavity. Comparison of experimental spectra with simulations shows the transmission peaks shift to shorter wavelengths for the twisted configuration. The shift is less than the half width at half maximum of a peak. Three peaks in the range of 480$500 \mathrm{~nm}$ have minimal intensity and maximal shift dispersion. However, all 14 points fit satisfactorily into the simulated dependence curve.

Figure 8(b) shows by dots and circles the experimental and calculated values of the SS, respectively. Analytical Eqs. (25) and (27) are shown by the solid curve and the dotted curve, respectively. The reflection phase shift leads to simultaneous displacement of all the points along the solid curve. The latter analytic curve is affected by the reflection phase shift only near Gooch-Terry maxima. The experimental spectrum contains no 
Gooch-Terry maxima. So in analytical equations the reflection anisotropy was ignored $\delta_{\mu}=0$.

The analytic formula (25) slightly overestimates the shift. There are several possible reasons for this distinction. First, the difference of PVA layer thicknesses on mirrors leads to some error. Second, weak reflection waves arising in the LC bulk produce the difference between the Berreman and the Jones SSs. The effective Jones RI (14) is commonly used for the TN LC where the helical pitch is much larger than the wavelength [21]. The formula (14) has been generalized $[61,64,87,88]$ for cholesteric LC where the helical pitch is of the same order with the wavelength. The wave vector for the Berreman method:

$$
q^{ \pm}= \pm \sqrt{k_{\varphi}^{2}+n^{2} k_{0}^{2} \pm k_{0} \sqrt{4 k_{\varphi}^{2} n^{2}+\left(2 n \delta n+\delta n^{2}\right)^{2} k_{0}^{2}}} .
$$

It gives the effective Berreman RI:

$$
n_{B}^{ \pm}= \pm \sqrt{n_{\varphi}^{2}+n^{2} \pm \sqrt{4 n_{\varphi}^{2} n^{2}+\left(2 n \delta n+\delta n^{2}\right)^{2}}} .
$$

The Berreman RI (32) and the Jones RI (14) contrast is evident in the graph scale despite the reasonable approximation $\varphi \ll$ $\delta \ll \sigma$.

\section{CONCLUDING REMARKS}

To the best of our knowledge, the Mauguin phase shift formula (13) has had only implicit and indirect confirmations in polarization measurements. Such measurements are, for example, the measurement of spectral positions of Gooch-Terry minima and the optical activity measurement of cholesteric LC [75]. As usual, in commercial TN cells TSS does not exceed $1 \mathrm{~nm}$ in accordance with Eq. (27). TSS is really hard to ascertain experimentally. For example, in the experimental spectra of TN-FPC with twist-homeotropic electric switching, the $o$-polarized SS, as reported in [58], was much higher than TSS Eq. (27). The crucial factor was reorientation of the parietal LC sublayers. They were not reoriented homeotropically up to the cell breakdown voltage.

The reported shift in TN-FPC can be observed directly without any polarizers. The required measurement accuracy is achieved due to multiple interference in the cavity. The main drawback of the presented scheme is that untwisted structure maintains a constant RI for the $o$-polarized light only. The $e$-polarized SS may be measured in the experimental scheme with the twist-planar to homogeneous-planar transition, which could be achieved using photoalignment material with reversible intermolecular bonds [89,90].

The avoided crossing SS caused by the mirror reflection mode coupling, the above-mentioned RSS, was described analytically and experimentally in the uniformly twisted structure. The analytical expression (26) for RSS near the
Gooch-Terry maximum looks much easier than the one published before [34]. A generalized Mauguin-Poincaré rolling cone method allowed us to solve the problem geometrically, independently of the Jones and Berreman matrix formalisms.

The SS of transmission peaks should not be confused with a frequency shift or a frequency conversion. In the stationary linear problem the light frequency is not converted. Also, we claim that the TSS characterizes not entirely the cavity but namely the twisted layer itself. The cavity just facilitates the measurement in that the twisted layer does not generate any transmission peaks. So what is actually shifted while twisting the layer? Obviously, the eigenwave phase is shifted when going out of the twisted layer. Therefore, the effective RI varies. This optical response can be measured without a cavity. For example, a polarization grating has considerable sensitivity to a minute change in RI, which permits the experimental confirmation of the described phenomenon.

The intermediate optical response presumption was formulated to determine the extreme values of the optical response of the complex medium. Despite the apparent evidence, this presumption may be violated. A well-known example is a composite of a few optical media with the inhomogeneity scale much smaller than the wavelength. Its resonant optical response may exceed maximal values for every component. This intermediate optical response violation for composites is explained by the Clausius-Mossotti contribution of spatial boundaries between the components [91,92]. Another intermediate optical response violation for a twisted anisotropic medium is shown to be due to the GP contribution. This tiny GP contribution should not be confused with the GP of the zerothorder adiabatic approximation. The last one is responsible for $\pi$-phase polarization conflict in the $\pi$-twisted LC cell [11]. A visual GP representation is speculated as the area covered by the polarization trajectory on PS. Contributions of TSS and RSS are related to areas of spherical rectangles and triangles.

The revealed tiny SS in TN-FPC exists in an arbitrary anisotropic chiral medium, not only in the TN layer. Without a doubt, a vast class of twist-polarization devices in highprecision engineering applications, such as multiplexers, 3D displays, optical tweezers, holographic data storage, and diffractive optics must be accounted for.

\section{ACKNOWLEDGMENTS}

This work was supported in part by Russian Foundation for Basic Research Grants No. 14-02-31248 and No. 15-0206924; Ministry of Education and Science of the Russian Federation under the Government program, Project No. 3.1276.2014/K; and the Ministry of Science and Technology of Taiwan under Grant No. NSC 103-2923-M-009-003-MY3 through an NSC-SB RAS joint project.
[1] L. M. Blinov, Structure and Properties of Liquid Crystals (Springer, Berlin, 2010).

[2] M. V. Berry, Proc. R. Soc. A Math. Phys. Eng. Sci. 392, 45 (1984).
[3] D. Chruscinski and A. Jamiolkowski, Geometric Phases in Classical and Quantum Mechanics, Progress in Mathematical Physics (Birkhäuser, Boston, 2004). 
[4] S. Pancharatnam, Proc. Indian Acad. Sci., Sec. A 44, 247 (1956)

[5] D. Bouwmeester, N. H. Dekker, F. E. V Dorsselaer, C. A. Schrama, P. M. Visser, and J. P. Woerdman, Phys. Rev. A 51, 646 (1995).

[6] D. N. Klyshko, Usp. Fiz. Nauk 36, 1005 (1993).

[7] L. Lu, J. D. Joannopoulos, and M. Soljačić, Nat. Photonics 8 , 821 (2014).

[8] B. Zhen, C. W. Hsu, L. Lu, A. D. Stone, and M. Soljačić, Phys. Rev. Lett. 113, 257401 (2014).

[9] M. Z. Hasan and C. L. Kane, Rev. Mod. Phys. 82, 3045 (2010).

[10] T. Bauer, P. Banzer, E. Karimi, S. Orlov, A. Rubano, L. Marrucci, E. Santamato, R. W. Boyd, and G. Leuchs, Science 347, 964 (2015).

[11] M. V. Vasnetsov, V. A. Pas'ko, and D. S. Kasyanyuk, Opt. Lett. 36, 2134 (2011).

[12] E. Hasman, G. Biener, A. Niv, and V. Kleiner, Prog. Opt. 47, 215 (2005).

[13] S. R. Nersisyan, N. V. Tabiryan, D. M. Steeves, and B. R. Kimball, Opt. Photonics News 21, 40 (2010).

[14] S. Pancharatnam, Proc. Indian Acad. Sci., Sec. A 41, 137 (1955).

[15] R. Simon, H. J. Kimble, and E. C. G. Sudarshan, Phys. Rev. Lett. 61, 19 (1988).

[16] P. F. McManamon, P. J. Bos, M. J. Escuti, J. Heikenfeld, S. Serati, H. Xie, and E. A. Watson, Proc. IEEE 97, 1078 (2009).

[17] P. Hariharan, Prog. Opt. 48, 149 (2005).

[18] S. Isaacs, F. Placido, and I. Abdulhalim, Appl. Opt. 53, H91 (2014).

[19] V. Y. Zyryanov, V. A. Gunyakov, S. A. Myslivets, V. G. Arkhipkin, and V. F. Shabanov, Mol. Cryst. Liq. Cryst. 488, 118 (2008).

[20] V. A. Gunyakov, M. N. Krakhalev, V. Y. Zyryanov, and V. F. Shabanov, Tech. Phys. Lett. 41, 86 (2015).

[21] Y.-C. Hsiao, C.-Y. Wu, C.-H. Chen, V. Y. Zyryanov, and W. Lee, Opt. Lett. 36, 2632 (2011).

[22] A. Vargas, M. del Mar Sánchez-López, P. García-Martínez, J. Arias, and I. Moreno, J. Appl. Phys. 115, 033101 (2014).

[23] A. Márquez, I. Moreno, J. Campos, and M. J. Yzuel, Opt. Commun. 265, 84 (2006).

[24] C. V. Mauguin, Bull. Soc. Fr. Mineral. 34, 71 (1911).

[25] J. S. Patel and Y. Silberberg, Opt. Lett. 16, 1049 (1991).

[26] R. C. Jones, J. Opt. Soc. Am. 31, 488 (1941).

[27] F. Abelès, Prog. Opt. 2, 249 (1963).

[28] M. Born and E. Wolf, Principles of Optics: Electromagnetic Theory of Propagation, Interference and Diffraction of Light (Cambridge University Press, Cambridge, UK, 1999).

[29] P. Yeh, A. Yariv, and C.-S. Hong, J. Opt. Soc. Am. 67, 423 (1977).

[30] P. Yeh, J. Opt. Soc. Am. 69, 742 (1979).

[31] P. Yeh and C. Gu, Optics of Liquid Crystal Displays (Wiley, New York, 1999).

[32] H. Gooch and C. Tarry, J. Phys. D: Appl. Phys. 8, 1575 (1975).

[33] R. M. A. Azzam and N. M. Bashara, Ellipsometry and Polarized Light (North-Holland, Amsterdam, 1977).

[34] Y. Ohtera, H. Yoda, and S. Kawakami, Opt. Quantum Electron. 32, 147 (2000).

[35] B. E. A. Saleh and M. C. Teich, Fundamentals of Photonics (Wiley, New York, 2007).

[36] S. Teitler and B. W. Henvis, J. Opt. Soc. Am. 60, 830 (1970).

[37] D. W. Berreman, J. Opt. Soc. Am. 62, 502 (1972).

[38] S. P. Palto, J. Exp. Theor. Phys. 92, 552 (2001).
[39] Z. Zhuang and J. S. Patel, Opt. Lett. 24, 1759 (1999).

[40] V. A. Belyakov, in New Developments in Liquid Crystals and Applications, edited by P. K. Choudhury, Materials Science Technology (Nova Science, New York, 2013), pp. 199-227.

[41] A. H. Gevorgyan, K. B. Oganesyan, G. A. Vardanyan, and G. K. Matinyan, Laser Phys. 24, 115801 (2014).

[42] I. V Timofeev, V. G. Arkhipkin, S. Y. Vetrov, V. Y. Zyryanov, and W. Lee, Opt. Mater. Express 3, 496 (2013).

[43] L. T. Song, J. He, H. L. Wang, Y. A. Han, and T. Li, Optoelectron. Lett. 8, 277 (2012).

[44] C. Zhang, N. Diorio, O. D. Lavrentovich, and A. Jákli, Nat. Commun. 5, 3302 (2014).

[45] A. D. Kiselev and V. G. Chigrinov, Phys. Rev. E 90, 042504 (2014).

[46] S. P. Palto, M. I. Barnik, A. R. Geivandov, I. V. Kasyanova, and V. S. Palto, Phys. Rev. E 92, 032502 (2015).

[47] H. Yoda, Y. Ohtera, O. Hanaizumi, and S. Kawakami, Opt. Quantum Electron. 29, 285 (1997).

[48] X. Zhu, Q. Hong, Y. Huang, and S.-T. Wu, J. Appl. Phys. 94, 2868 (2003).

[49] C. R. Fernández-Pousa, I. Moreno, N. Bennis, and C. GómezReino, J. Opt. Soc. Am. A 17, 2074 (2000).

[50] S. Stallinga, J. Appl. Phys. 86, 4756 (1999).

[51] H. Poincaré, M. Lamotte, and D. Hurmuzescu, Théorie Mathématique de La Lumière II: Nouvelles Études Sur La Diffraction-Théorie de La Dispersion de Helmholtz, Leçons Professées Pendant Le Premier Semestre 1891-1892 (G. Carré, 1892).

[52] Y.-T. Lin, W.-Y. Chang, C.-Y. Wu, V. Y. Zyryanov, and W. Lee, Opt. Express 18, 26959 (2010).

[53] S. Y. Vetrov and A. V. Shabanov, J. Exp. Theor. Phys. 93, 977 (2001).

[54] V. F. Shabanov, S. Y. Vetrov, and A. V. Shabanov, Optics of Real Photonic Crystals (Russian Academy of Sciences, Novosibirsk, 2005) [in Russian].

[55] A. Baldycheva, V. A. Tolmachev, K. Berwick, and T. S. Perova, Nanoscale Res. Lett. 7, 387 (2012).

[56] J. Schmidtke, W. Stille, and H. Finkelmann, Phys. Rev. Lett. 90, 083902 (2003).

[57] T. Matsui, M. Ozaki, and K. Yoshino, Phys. Rev. E 69, 061715 (2004).

[58] I. V. Timofeev, Y. T. Lin, V. A. Gunyakov, S. A. Myslivets, V. G. Arkhipkin, S. Y. Vetrov, W. Lee, and V. Y. Zyryanov, Phys. Rev. E 85, 011705 (2012).

[59] V. G. Arkhipkin, V. A. Gunyakov, S. A. Myslivets, V. P. Gerasimov, V. Y. Zyryanov, S. Y. Vetrov, and V. F. Shabanov, J. Exp. Theor. Phys. 106, 388 (2008).

[60] I. V. Timofeev and S. Y. Vetrov, Bull. Russ. Acad. Sci.: Phys. 78, 1308 (2014).

[61] C. W. Oseen, Trans. Faraday Soc. 29, 883 (1933).

[62] I. Abdulhalim, Opt. Lett. 31, 3019 (2006).

[63] V. A. Belyakov, Mol. Cryst. Liq. Cryst. 612, 81 (2015).

[64] V. A. Belyakov, Diffraction Optics of Complex-Structured Periodic Media (Springer, New York, 1992).

[65] H. A. Macleod, Thin-Film Optical Filters, 4th ed., Series in Optics and Optoelectronics (CRC Press, Boca Raton, FL, 2010).

[66] B. Gralak, M. Lequime, M. Zerrad, and C. Amra, J. Opt. Soc. Am. A 32, 456 (2015).

[67] S. P. Palto, N. M. Shtykov, B. A. Umanskii, and M. I. Barnik, J. Appl. Phys. 112, 013105 (2012). 
[68] M. I. Rabinovich and D. I. Trubetskov, Oscillations and Waves: In Linear and Nonlinear Systems, Mathematics and Its Applications (Springer, Berlin, 1989).

[69] A. Messiah, Quantum Mechanics (Dover, Mineola, NY, 1999).

[70] H. G. Jerrard, J. Opt. Soc. Am. 44, 634 (1954).

[71] D. H. Goldstein, Polarized Light, 3rd ed. (CRC Press, Boca Raton, FL, 2010).

[72] X. Yi, Y. Liu, X. Ling, X. Zhou, Y. Ke, H. Luo, S. Wen, and D. Fan, Phys. Rev. A 91, 023801 (2015).

[73] V. Lakshminarayanan, M. L. Calvo, and T. Alieva (eds.), Mathematical Optics (CRC Press, Boca Raton, FL, 2012).

[74] A. A. Muller, P. Soto, D. Dascalu, D. Neculoiu, and V. E. Boria, IEEE Microwave Wireless Compon. Lett. 21, 286 (2011).

[75] S. Chandrasekhar, Liquid Crystals (Cambridge University Press, Cambridge, UK, 1992).

[76] W. A. Shurcliff, Polarized Light: Production and Use (Harvard University Press, Cambridge, MA, 1962).

[77] H. M. Jeffery, Q. J. Pure Appl. Math. 19, 44 (1883).

[78] K. Y. Bliokh, A. Niv, V. Kleiner, and E. Hasman, Nat. Photonics 2, 748 (2008).

[79] I. M. Gelfand, R. A. Minlos, and G. Cummins, Representations of the Rotation and Lorentz Groups and Their Applications (Martino, Eastford, CT, 2012).

[80] J. H. Conway and D. Smith, On Quaternions and Octonions (A K Peters/CRC Press, Boca Raton, FL, 2003).

[81] G. A. Korn and T. M. Korn, Mathematical Handbook for Scientists and Engineers: Definitions, Theorems, and Formulas for Reference and Review, 2nd revised ed., Dover Civil and Mechanical Engineering (Dover, Mineola, NY, 2000).

[82] D. Hilbert and S. Cohn-Vossen, Geometry and the Imagination (Chelsea, New York, 1952).

[83] S. P. Palto, L. M. Blinov, M. I. Barnik, V. V. Lazarev, B. A. Umanskii, and N. M. Shtykov, Crystallogr. Rep. 56, 622 (2011).

[84] J. Cognard, Alignment of Nematic Liquid Crystals and Their Mixtures: A Special Issue of the Journal Molecular Crystals and Liquid Crystals, Topics in Chemical Engineering (Routledge, New York, 1982).

[85] J. E. Proust, L. Ter-Minassian-Saraga, and E. Guyon, Solid State Commun. 11, 1227 (1972).

[86] V. S. Sutormin, M. N. Krakhalev, O. O. Prishchepa, W. Lee, and V. Y. Zyryanov, Opt. Mater. Express 4, 810 (2014).

[87] H. de Vries, Acta Crystallogr. 4, 219 (1951).

[88] E. I. Kats, Sov. Phys. J. Exp. Theor. Phys. 32, 1004 (1971).

[89] V. G. Chigrinov, V. M. Kozenkov, and H. S. Kwok, Photoalignment of Liquid Crystalline Materials: Physics and Applications (Wiley, New York, 2008).

[90] V. Mikulich, A. Murauski, A. Muravsky, V. Agabekov, and V. Bezruchenko, J. Soc. Inf. Disp. 22, 199 (2014).

[91] A. Sihvola, Electromagnetic Mixing Formulae and Applications, IEEE Electromagnetic Waves Series Vol. 47 (Institution of Engineering and Technology, Stevenage, UK, 1999).

[92] T. G. Mackay, A. Lakhtakia, D. M. Richardson, and A. Ricciardi, Electromagnetic Anisotropy and Bianisotropy: A Field Guide (World Scientific, Singapore, 2009). 